\title{
Upcoming conferences in 1996
}

May 17-19, 1996

Fourth International Congress on Physical Education and Sport, Komotini, Greece. Contact: Democritus University of Thrace, Department of Physical Education and Sport Science, Secretariate 4th I.C.P.E.S., Komotini, 69100, Greece.

May 28-June 1, 1996

American Association on Mental Retardation, Annual Conference, San Antonio, Texas. Contact: AAMR, Tel.: $1800 / 4243688$.

June 4-8, 1996

Eighth Annual Intensive Postsecondary LD Training Institute for Postsecondary Personnel. Newport, Rhode Island. Contact: Stan Shaw or Patricia L. Anderson, Tel.: 860/486 0163 or 860/486 0273.

June 6-8, 1996

Quality of Life: An International Conference for Families and Professionals on Developmental and Related Disabilities. Toronto, Ontario, Canada. Contact: Quality of Life Conference, Surrey Place Centre, c/o Continuing Education, 150 College Street, Room 121, Toronto, Ontario, Canada M5S 1A8.

June 11-13, 1996

Seventeenth Annual National Educational Computing Conference. Minneapolis, Minnesota. Contact: NECC'96, 2665 Long Lake Road, Suite 250, Roseville, MN 55113-2535: Tel.: 612/638 8764; fax: +1 612 6388769 .

\section{June 24-28, 1996}

Aspen Summer Institute-Enhancing Special Education for the 21st Century: Paradigms, Programs, and Practices. Aspen, Colorado. Contact: Dr. J. Frederick West, Institute for Educational Development and Training, P.O. Box 99515, Raleigh, NC 27624-9515: Tel.: +1 919847 2242; fax: +1 9198471073.

July 8-13, 1996

Tenth World Conference, International Association for the Scientific Study of Intellectual Disability. Helsinki, Finland. Contact: Dr. Trevor R. Parmenter, Unit for Community Integration Studies, School of Education, Macquarie University. North Ryde NSW 2109, Australia; Tel.: +61 2850 8706; fax: +61 2 850 8674; email: T.Palm@ted.educ.mq.edu.au.

July 9-13, 1996

Summer Institute on Inclusive Schools and Classrooms: Making It Happen. Montreal, Quebec, Canada. Contact: Joan Spence, McGill University, CEL, 3724 McTavish St., Montreal Quebec, Canada H3A 1Y2; Tel.: +1 514398 7044; fax: +1 514398 8260; email: leadership@cel.lan.mcgill.ca. 
July 10-13, 1996

Autism Society of America's 1996 National Conference: Open Minds, Open Doors. Milwaukee, Wisconsin. Contact: +1 414427 9345; fax: +14144279395.

August 7-10, 1996

Seventh Biennial Conference of the International Society for Augmentative and Alternative Communication. Vancouver, British Columbia, Canada. Contact: ISACC'96 Secretariat, c/o Venue West, 645-375 Water St., Vancouver, BC V6B 5C6; Tel.: +1604 681 5226; fax: +16046812503. 